Martin Picard, Darmyn Ritchie, Melissa M. Thomas, Kathryn J.

Wright, and Russell T. Hepple

\title{
Alterations in intrinsic mitochondrial function with aging are fiber type-specific and do not explain differential atrophy between muscles
}

Published in:

Aging Cell (2011) 10, pp1047-1055.

Available under a Creative Commons Attribution NonCommercial licence (v3.0).

doi: 10.1111/j.1474-9726.2011.00745.x http://onlinelibrary.wiley.com/doi/10.1111/j.1474-

9726.2011.00745.x/full 


\section{Alterations in intrinsic mitochondrial function with aging are fiber type-specific and do not explain differential atrophy between muscles}

\author{
Martin Picard, ${ }^{1,2}$ Darmyn Ritchie, ${ }^{3}$ Melissa M. Thomas, ${ }^{3}$ \\ Kathryn J. Wright ${ }^{3}$ and Russell T. Hepple ${ }^{1,2}$ \\ ${ }^{1}$ Department of Kinesiology, McGill University, Montreal, Quebec, Canada \\ H3A 1 A 1 \\ ${ }^{2}$ Department of Medicine, McGill University, Montreal, Quebec, Canada \\ H3A 1 A1 \\ ${ }^{3}$ Muscle \& Aging Laboratory, Faculty of Kinesiology, University of Calgary, \\ Calgary, Alberta, Canada T2N 1N4
}

\section{Summary}

To determine whether mitochondrial dysfunction is causally related to muscle atrophy with aging, we examined respiratory capacity, $\mathrm{H}_{2} \mathrm{O}_{2}$ emission, and function of the mitochondrial permeability transition pore (mPTP) in permeabilized myofibers prepared from four rat muscles that span a range of fiber type and degree of age-related atrophy. Muscle atrophy with aging was greatest in fast-twitch gastrocnemius (Gas) muscle ( $-38 \%)$, intermediate in both the fast-twitch extensor digitorum longus (EDL) and slow-twitch soleus (Sol) muscles (-21\%), and non-existent in adductor longus ( $\mathrm{AL}$ ) muscle $(+\mathbf{4 7 \%} \%$ ). In contrast, indices of mitochondrial dysfunction did not correspond to this differential degree of atrophy. Specifically, despite higher protein expression for oxidative phosphorylation (oxphos) system in fast Gas and EDL, state III respiratory capacity per myofiber wet weight was unchanged with aging, whereas the slow Sol showed proportional decreases in oxphos protein, citrate synthase activity, and state III respiration. Free radical leak $\left(\mathrm{H}_{2} \mathrm{O}_{2}\right.$ emission per $\mathrm{O}_{2}$ flux) under state III respiration was higher with aging in the fast Gas, whereas state II free radical leak was higher in the slow AL. Only the fast muscles had impaired MPTP function with aging, with lower mitochondrial calcium retention capacity in EDL and shorter time to MPTP opening in Gas and EDL. Collectively, our results underscore that the age-related changes in muscle mitochondrial function depend largely upon fiber type and are unrelated to the severity of muscle atrophy, suggesting that intrinsic changes in mitochondrial function are unlikely to be causally involved in aging muscle atrophy.

Key words: aging; sarcopenia; skeletal muscle.

\section{Introduction}

Impairment in mitochondrial function is posited to play an important role in the aging-related loss of muscle mass known as sarcopenia (Hiona \& Leeuwenburgh, 2008; Hepple, 2011). Three primary functions of the mitochondrion in aging muscle are likely relevant to this process: ATP pro-

\section{Correspondence}

Russell T. Hepple, Critical Care Division, Royal Victoria Hospital, 687 Pine Ave W, Room L3.01, Montreal, QC, Canada H3A 1A1. Tel.: 514 934-1934, ext. 35509; fax: 514 398-4186; e-mail: russell.hepple@mcgill.ca

Accepted for publication 6 September 2011 duction, reactive oxygen species (ROS) production, and the function of the apoptosis-regulating mitochondrial permeability transition pore (mPTP) (Hepple, 2011). A reduced ATP generating capacity may shift signaling pathways toward catabolism secondary to the activation of 5'adenosine monophosphate-activated protein kinase (AMPK) (Tong et al., 2009), and elevations of ROS and impaired mPTP function can cause muscle atrophy by activating autophagy and proteasome pathways (McClung et al., 2008) and mitochondrial-mediated pathways of apoptosis (Marzetti et al., 2010), respectively.

Consistent with the hypothesis that mitochondrial changes are important in sarcopenia, numerous studies have identified increases in ROS production (Capel et al., 2005; Muller et al., 2007; Chabi et al., 2008) and altered function of the mPTP (Chabi et al., 2008; Seo et al., 2008; Picard et al., 2010) in aging muscle. In addition, several mechanisms have been proposed to account for an accumulation of mitochondria with impaired function in aging muscle, including mitochondrial DNA damage (Wanagat et al., 2001; Hiona et al., 2010), reduced mitochondrial protein turnover resulting from both reduced mitochondrial degradation and reduced synthesis of new mitochondria (Rooyackers et al., 1996; Baker et al., 2006; Chabi et al., 2008; Ljubicic \& Hood, 2009), and mitochondrial iron accumulation leading to an exacerbation of mitochondrial oxidative stress (Seo et al., 2008).

Despite the accumulated evidence in favor of mitochondrial dysfunction playing an important role in aging muscle atrophy, recent evidence suggests this issue requires further study. Firstly, we showed that one of the most widely studied manifestations of accumulated mtDNA damage on muscle mitochondrial phenotype, which being a selective deficiency in the activity of complex IV (cytochrome c oxidase; COX) in muscle fibers (Wanagat et al., 2001; Bua et al., 2006), occurs with very low frequency $(<0.2 \%)$ even in severely atrophied aging muscle, and COX deficient myofibers are not particularly atrophied compared to other myofibers in aged muscle (Rowan et al., 2011). In addition, we recently showed that mitochondrial isolation, which is one of the primary methods used to interrogate mitochondrial function in skeletal muscle, including many studies in aging muscle (Desai et al., 1996; Capel et al., 2005; Muller et al., 2007; Chabi et al., 2008; Seo et al., 2008; Figueiredo et al., 2009; Gouspillou et al., 2010), markedly alters the function of mitochondria compared to permeabilized myofibers, a preparation that preserves the mitochondrial structure and allows study of the whole mitochondrial population (Picard et al., 2011). Further to this point, mitochondrial isolation markedly exaggerated the severity of mitochondrial dysfunction in severely atrophied aging muscle (Picard et al., 2010).

On the basis of this recent evidence questioning the magnitude of mitochondrial dysfunction in aging muscle, the current study sought to provide a more comprehensive test of the hypothesis that the intrinsic impairment in mitochondrial function causes aging muscle atrophy. To achieve this objective, we employed several novel features in the design of these experiments. Firstly, we examined four muscles that not only span extremes of fast [gastrocnemius (Gas) and extensor digitorum longus (EDL)] vs. slow [soleus (Sol) and adductor longus (AL)] fiber type profile (Armstrong \& Phelps, 1984), but also exhibit a wide range of atrophy with aging (R.T. Hepple, unpublished observations). Secondly, we exam- 
ined key indices of mitochondrial function implicated in sarcopenia including respiratory capacity, ROS emission, and function of the MPTP (Hepple, 2011). Finally, all of these measurements were performed in permeabilized myofibers to avoid potential artifacts secondary to mitochondrial isolation (Picard et al., 2010, 2011).

Our results revealed that alterations in intrinsic mitochondrial function with aging were relatively mild and segregated principally as a function of muscle fiber type composition. In particular, whereas the severity of muscle atrophy was greatest in fast-twitch Gas muscle, intermediate in fasttwitch EDL and in slow-twitch Sol muscles, and non-existent in slowtwitch AL muscle, impaired respiratory function in relation to protein abundance of the oxidative phosphorylation system was specific to fasttwitch muscles and did not differ between Gas and EDL, despite the large differences in atrophy between these muscles. On the other hand, elevated ROS production with aging was unrelated to fiber type but like respiration, did not explain differential atrophy with aging. Finally, altered function of the mPTP was specific to fast-twitch muscles with aging where the degree of mPTP dysfunction did not correspond to the large differences in degree of atrophy between Gas and EDL muscles. Collectively, therefore, the results show that the changes in intrinsic mitochondrial function with aging in skeletal muscle depend upon the fiber type examined and can differ between muscles with similar fiber type and between muscles with similar atrophy, where no single functional index explains the differential atrophy susceptibility between muscles.

\section{Results}

\section{Muscle atrophy severity}

As shown in Fig. 1, age-related muscle atrophy was greatest in the fasttwitch Gas muscle, intermediate in the fast-twitch EDL and slow-twitch Sol muscles, and non-existent in the AL muscle. In fact, the AL exhibited a striking of $47 \%$ increase in mass between young adulthood (YA) and senescence (SEN). To our knowledge, this is the first time any muscle has been reported to hypertrophy in very advanced age. Overall, these results demonstrate the considerable heterogeneity in age-related atrophy between muscles of similar fiber type composition and also show one example where the severity of atrophy is remarkably similar between a fast vs. a slow muscle, underscoring that atrophy severity is not simply a function of fiber type composition.

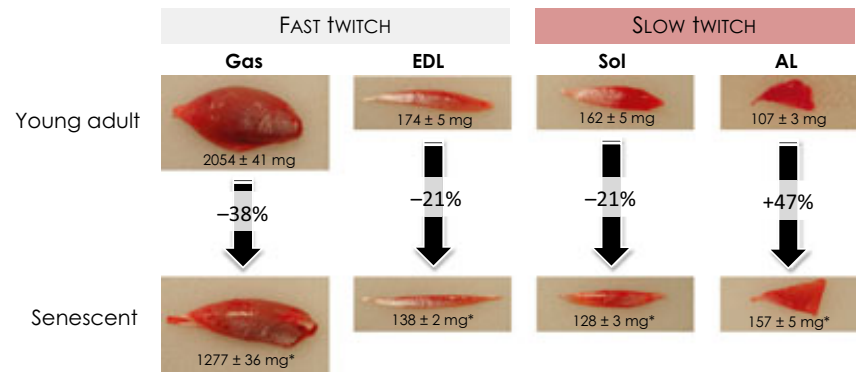

Fig. 1 Photographs of four hindlimb muscles from young adult (YA) and senescent Fischer $344 \times$ Brown Norway F1 rats demonstrating the considerable heterogeneity in severity of muscle atrophy with aging. Muscle atrophy differs both between muscles of different fiber type composition and between muscles of similar fiber type composition. Specifically, shown are examples of widely divergent severity of atrophy between fast Gastrocnemius (Gas) and fast extensor digitorum longus (EDL) muscles, and between slow soleus (Sol) and slow adductor longus ( $A L$ ) muscles. In addition, shown is an example of a fast and a slow muscle with the same severity of atrophy (fast EDL vs. slow Sol). $n=10$ per age group, per muscle; values are means \pm SEM; ${ }^{*} P<0.001$ vs. YA.

\section{Oxidative phosphorylation system subunit protein expression and mitochondrial enzyme activity}

Figure 2 (mean data) and Fig. S1 (Supporting information) (representative blots) shows the results of our analysis of the protein expression levels of representative subunits of each of the oxidative phosphorylation complexes. To provide for easy comparisons between muscles and ages, and also to permit comparison of the stoichiometry between complexes, all values have been normalized to the protein amount seen in complex I of the Gas muscle in YA animals. Our results showed that both fast-twitch Gas (Fig. 2A) and EDL (Fig. 2B) muscles had an increased expression of components of the oxidative phosphorylation system. In contrast, the slow-twitch Sol muscle had a lower expression of a representative subunit in complexes I and IV (Fig. 2C). There were no changes seen in any subunit examined in the AL muscle (Fig. 2D). Collectively, this analysis shows that the mitochondrial oxidative phosphorylation system protein content is elevated in aged fast-twitch muscle and maintained (AL) or marginally reduced (Sol) in aged slow-twitch muscle. As we have previously seen only a $10 \%$ lower total protein concentration per mg of muscle between YA and SEN (Hepple et al., 2005), the differences (or lack thereof) in oxidative phosphorylation protein content per mg protein between age groups largely reflect levels in the whole muscle (i.e., indicative of higher oxidative phosphorylation protein levels per muscle mass in the fast muscles with aging).

To obtain an index of mitochondrial content across muscles and with aging, we measured the biochemical activity of the mitochondrial matrix enzyme citrate synthase (CS) (Table 1) and the mitochondrial cristae membrane enzyme COX (Table 2). Consistent with previous data in rat muscles (Delp \& Duan, 1996), CS in YA was relatively similar across all four muscles, despite their varied fiber type composition. In contrast to the higher protein content for the oxidative phosphorylation system, the CS analyses revealed no change in mitochondrial content with aging in the fast-twitch muscles, and a selective reduction in CS activity in slowtwitch Sol $(-29 \%)$ muscle. The same was observed for COX activity. The discrepancy between the increase in the cristae-embedded electron transport chain protein abundance and the unchanged matrix-located CS activity in the fast muscles suggests compartment-specific changes in mitochondrial proteins with aging.

\section{Mitochondrial respiratory capacity}

Figure 3 shows the main differences observed as a function of age in the respirometry experiments. State III respiration - using complex I (glutamate, malate) and complex II (succinate) substrates - expressed per myofiber bundle wet weight was lower with aging only in the slow-twitch Sol muscle (Fig. 3A). After normalizing for CS activity to obtain an index of intrinsic mitochondrial respiratory capacity, the lower state III respiratory capacity in Sol with aging was abrogated and no other muscle demonstrated evidence of a mitochondrial-specific respiratory impairment when examined in this manner (Fig. 3B). However, the higher electron transport chain protein content that we report above in fast-twitch muscles suggests lower intrinsic electron transport chain function in fast muscles with aging, necessitating a higher protein content to achieve the same muscle mass-specific respiratory capacity.

As complex IV is often preferentially reduced with aging (Hepple et al., 2005; Navarro \& Boveris, 2007), we also examined the respiratory capacity in each muscle after the addition of the artificial electron donor, $\left[N, N, N^{\prime}, N^{\prime}\right.$-tetramethyl-p-phenylenediamine (TMPD)], which donates electrons directly to complex IV, thereby permitting quantification of complex IV respiratory capacity independent of upstream electron 

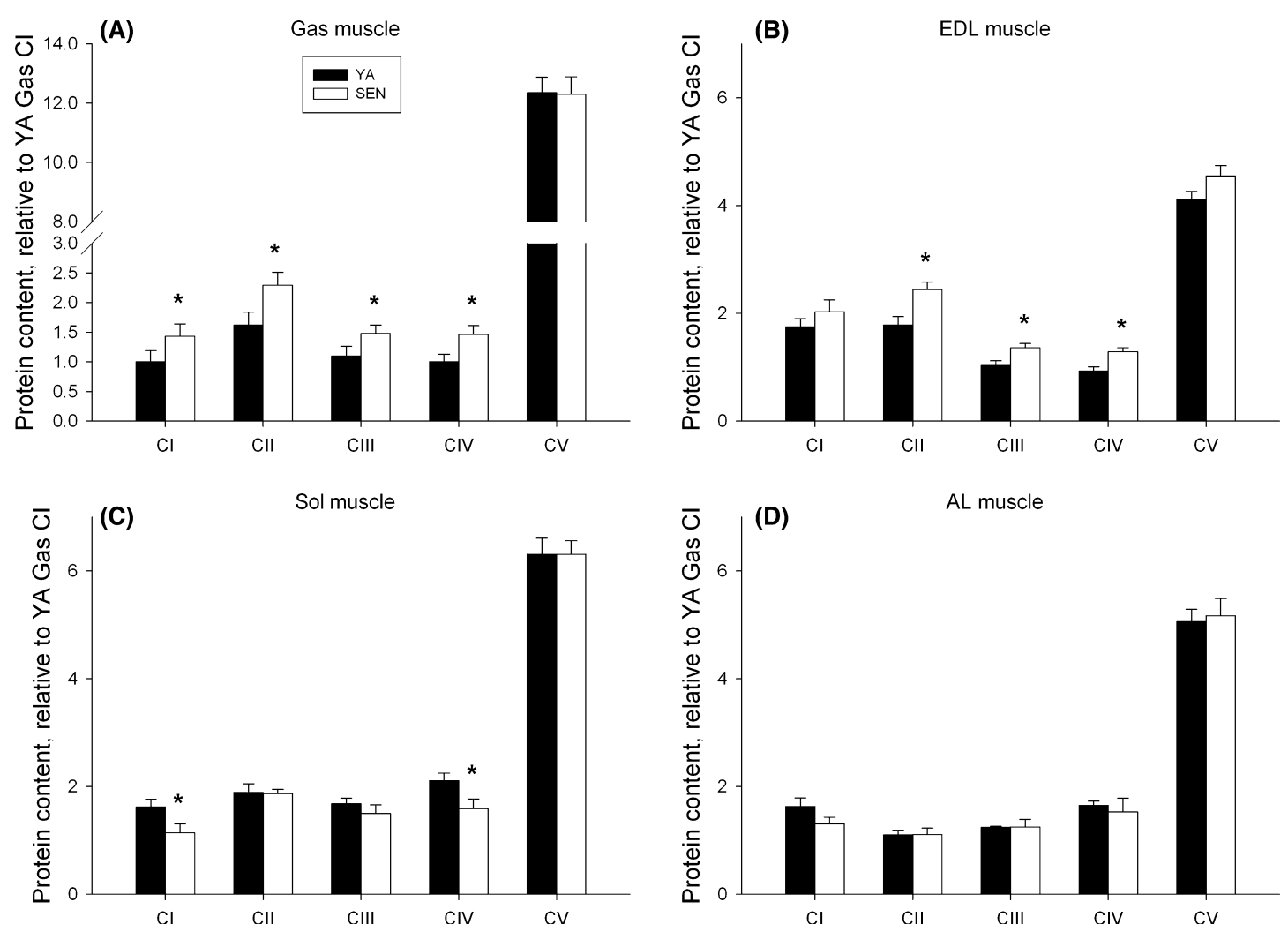

Fig. 2 Protein levels measured by Western Blot of representative subunits of each of the mitochondrial oxidative phosphorylation complexes in homogenates from gastrocnemius (Gas; A), extensor digitorum longus (EDL; B), soleus (Sol; C), and adductor longus (AL; D) muscles of young adult (YA) and senescent (SEN) rats. Data are expressed relative to $\mathrm{Cl}$ protein content for YA mGas. Antibodies used are specific to complex I subunit NDUFB8 (CI), complex II subunit $30 \mathrm{kDa}$ (CII), complex III subunit core 2 (CIII), complex IV subunit 1 (CIV), and complex V subunit alpha (CV). Representative Western blots are shown in Fig. S1 (Supporting information). $n=8$ animals per age group for each muscle except $\mathrm{AL}$ (YA: $n=4$; $\operatorname{SEN} n=5$ ); values are means $\pm \mathrm{SEM}$; $P<0.05$ vs. YA.

Table 1 Citrate synthase activity

\begin{tabular}{lllll}
\hline Age & Gas & EDL & Sol & AL \\
\hline YA & $14.1 \pm 1.6$ & $16.4 \pm 1.5$ & $18.9 \pm 1.3$ & $17.9 \pm 1.1$ \\
SEN & $16.2 \pm 2.2$ & $16.6 \pm 1.1$ & $13.4 \pm 1.2^{*}$ & $17.1 \pm 1.5$ \\
\hline
\end{tabular}

Measurements performed in permeabilized myofiber bundles used for respirometry, $n=8$ per group. Units are $\mu \mathrm{mol} \mathrm{min}^{-1} \mathrm{~g}^{-1}$ wet weight. Values are means $\pm \mathrm{SE} ; n=8$ per group.

$A L$, adductor longus; EDL, extensor digitorum longus; SEN, senescent; Sol, soleus; YA, young adult.

${ }^{*} P<0.05$ vs. $Y$ A.

Table 2 Cytochome coxidase activity

\begin{tabular}{llrrl}
\hline Age & Gas & \multicolumn{1}{l}{ EDL } & \multicolumn{1}{l}{ Sol } & \multicolumn{1}{l}{ AL } \\
\hline YA & $75 \pm 12$ & $90 \pm 3$ & $110 \pm 4$ & $120 \pm 4$ \\
SEN & $91 \pm 11$ & $102 \pm 8$ & $98 \pm 4^{*}$ & $125 \pm 3$ \\
\hline
\end{tabular}

Measurements performed in permeabilized myofiber bundles used for respirometry, $n=8$ per group. Units are $\mu \mathrm{mol} \min ^{-1} \mathrm{~g}^{-1}$ wet weight. Values are means $\pm \mathrm{SE}, n=6-8$ per group.

$\mathrm{AL}$, adductor longus; EDL, extensor digitorum longus; Sol, soleus; SEN, senescent; YA, young adult.

$\star P=0.06$. transport system components. As our results show, TMPD-driven respiration was lower only in the fast-twitch Gas and in the slow-twitch Sol (Fig. 3C). Given the lower expression of a subunit of complex IV in aged Sol muscle, the lower TMPD-driven respiration in Sol muscle is likely due to less protein rather than complex IV dysfunction per se. Other indices of mitochondrial respiratory function were also relatively well preserved across muscles with aging (Figs S2 and S3).

\section{$\mathrm{H}_{2} \mathrm{O}_{2}$ emission and endogenous antioxidant enzyme activities}

The amount of $\mathrm{H}_{2} \mathrm{O}_{2}$ emission per $\mathrm{O}_{2}$ flux - an index of free radical leak tended to be elevated in both fast muscles under state II conditions and was significantly elevated in Gas under state III conditions. Whereas we did not detect significant changes in free radical leak in slow Sol with aging, the $\mathrm{AL}$ had a higher $\mathrm{H}_{2} \mathrm{O}_{2}$ emission during state II driven respiration (Fig. 4).

Manganese superoxide dismutase (MnSOD) activity was elevated with aging only in the fast-twitch Gas muscle (Fig. 5A). Glutathione peroxidase (GPx) activity was elevated in fast-twitch Gas muscle and unchanged in fast-twitch EDL muscle (Fig. 5B). In contrast, GPx activity was lower in both slow-twitch Sol and AL muscles. Similarly to GPx, catalase (Cat) activity was elevated with aging in both fasttwitch Gas and EDL muscles (Fig. 5C). However, whereas Cat activity was lower in slow-twitch Sol muscle, it was elevated in the slowtwitch AL muscle. Generally, therefore, neither changes in ROS gen- 

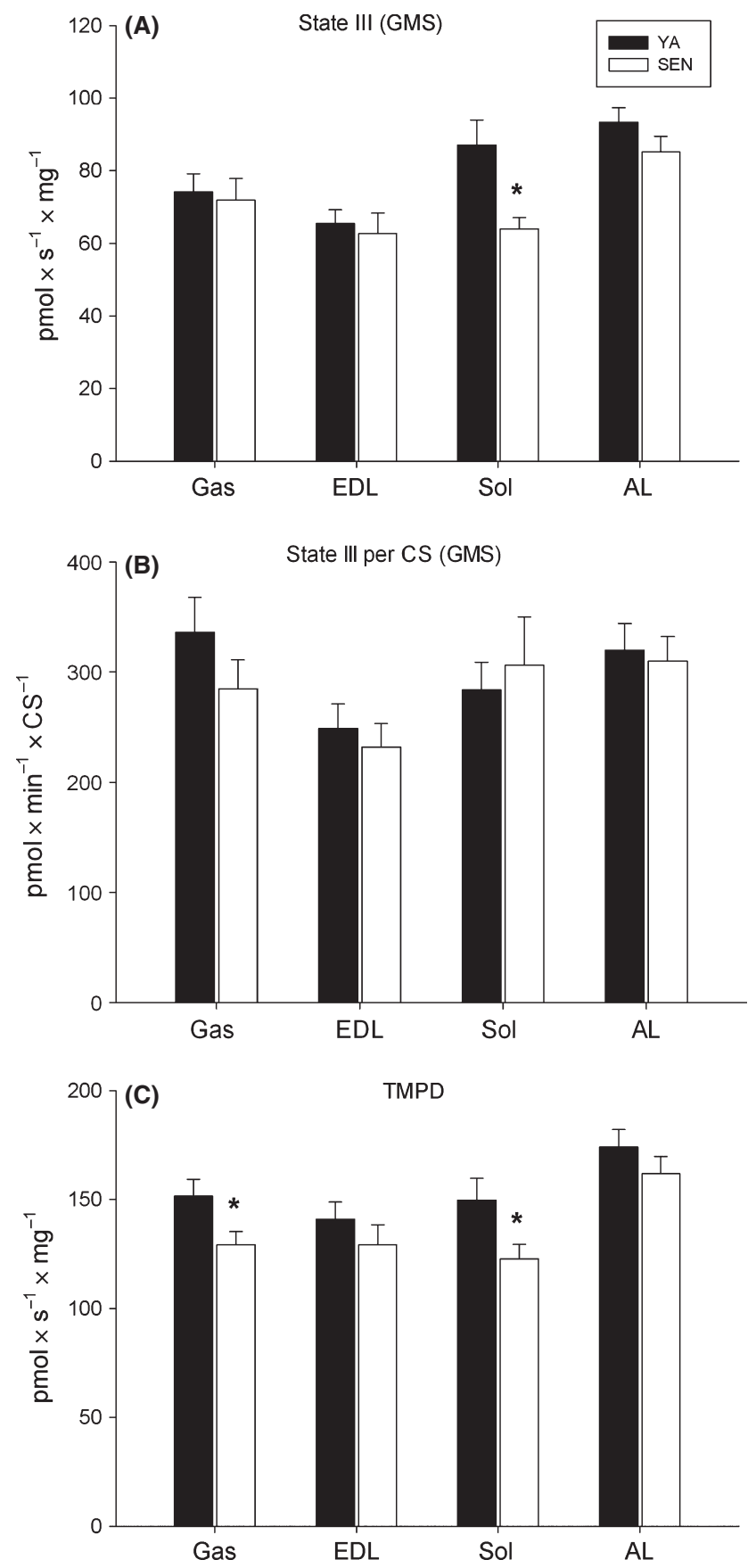

Fig. 3 Mitochondrial respiratory capacity measured by high-resolution oxygraphy in permeabilized myofibers of gastrocnemius (Gas), extensor digitorum longus $(E D L)$, soleus (Sol), and adductor longus $(A L)$ muscles of young adult $(Y A)$ and senescent (SEN) rats. (A) Mitochondrial oxygen consumption in the presence of glutamate, malate, and succinate (GMS) and adenosine di-phosphate (State III) normalized per fiber bundle wet weight. (B) State III GMS oxygen consumption normalized per international units of citrate synthase activity measured on individual fiber bundles. (C) Oxygen consumption by complex IV per wet weight after the addition of the artificial electron donor TMPD $\left(N, N, N^{\prime}, N^{\prime}\right.$-tetramethyl-pphenylenediamine). $n=8$ per age group for each muscle; values are means \pm SEM; ${ }^{*} P<0.05$ vs. YA.

eration nor adaptations in endogenous antioxidant defense systems corresponded to the severity of muscle atrophy with aging. Additional measures made in the $\mathrm{H}_{2} \mathrm{O}_{2}$ experiments are shown in Fig. $\mathrm{S} 4$
(Supporting information) and underscore the relatively minor changes observed with aging.

\section{Mitochondrial permeability transition pore function}

Figure 6 shows the results of our assessments of the function of the MPTP in response to $\mathrm{a} \mathrm{Ca}{ }^{2+}$ challenge. These experiments showed that the $\mathrm{Ca}^{2+}$ retention capacity $(C R C)$, after normalizing for a marker of mitochondrial content (CS activity), was lower with aging only in the fast-twitch EDL muscle (Fig. 6A). The time to MPTP opening was significantly reduced only in the fast-twitch Gas and EDL muscles (Fig. 6B). Despite significant muscle atrophy in slow-twitch Sol muscle, equal in magnitude to the fasttwitch EDL, there were no changes in MPTP function in the Sol muscle with aging. Similarly, there were no changes in mPTP function with aging in the AL muscle. As such, changes in MPTP function were specific to fasttwitch muscle and did not correspond to the severity of muscle atrophy with aging.

\section{Discussion}

The purpose of this study was to test the hypothesis that the mitochondrial dysfunction causes age-related muscle atrophy. To meet this objective, we examined three indices of mitochondrial function implicated in aging muscle atrophy and employed a unique study design involving comparisons between four muscles representing extremes of fast vs. slow fiber type composition and which exhibit a markedly different degree of atrophy with aging. If mitochondrial dysfunction is causally related to age-related muscle atrophy, we would expect that the severity of mitochondrial dysfunction should be greatest in the largely fast-twitch Gas muscle (38\% atrophy with aging), intermediate in the largely fast-twitch EDL and largely slow-twitch Sol muscles (21\% degree of atrophy with aging in both muscles), and non-existent in the slow-twitch AL muscle (hypertrophies $47 \%$ with aging). In contrast to our hypothesis, the results showed that the nature of alterations in mitochondrial function in aging muscles segregated principally as a function of fiber type composition. Specifically, the fast-twitch Gas and EDL muscles exhibited signs of impaired mitochondrial respiratory capacity relative to the abundance of oxidative phosphorylation system proteins, elevated $\mathrm{H}_{2} \mathrm{O}_{2}$ emission, and an impaired function of the MPTP that suggests greater apoptotic susceptibility in the aged fast-twitch muscles. On the other hand, the slow-twitch Sol muscle exhibited a mild reduction in muscle respiratory capacity consistent with the lower indices of mitochondrial content in this muscle with aging (i.e., no mitochondrial respiratory defect per se), and the slow AL exhibited an elevated $\mathrm{H}_{2} \mathrm{O}_{2}$ emission under state II conditions. We conclude that the atrophy of aging skeletal muscle is associated with fiber type-specific changes in canonical indices of the intrinsic function of mitochondria, where no one index of function predicts the severity of atrophy between muscles.

\section{Mitochondrial dysfunction and sarcopenia}

A progressive reduction in muscle mass is one of the hallmarks of normal aging and when it becomes severe it contributes to a marked increase in the risk of falls, mobility impairment, and physical frailty in the elderly (Cruz-Jentoft et al., 2010). Despite the abundance of evidence using isolated mitochondria finding support for a role for mitochondrial dysfunction in atrophy of aging muscle (Chabi et al., 2008; Seo et al., 2008; Hiona et al., 2010), no prior study has systematically determined whether the severity of mitochondrial dysfunction parallels the degree of atrophy with aging. Such evidence could go a long ways toward proving or dis- 

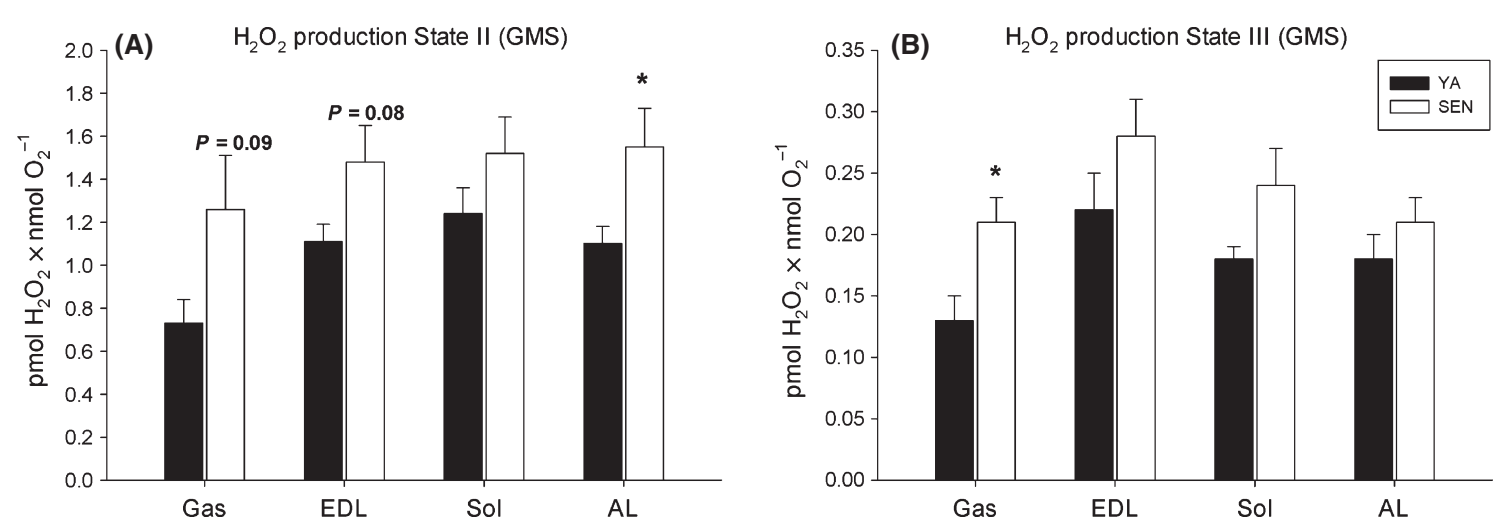

Fig. 4 Mitochondrial $\mathrm{H}_{2} \mathrm{O}_{2}$ release per unit oxygen flux (free radical leak) measured with Amplex Red from permeabilized myofibers of gastrocnemius (Gas), extensor digitorum longus (EDL), soleus (Sol), and adductor longus (AL) muscles of young adult (YA) and senescent (SEN) rats. (A) state II conditions with glutamate, malate and succinate (GMS); (B) state III conditions with GMS and 1 mM adenosine di-phosphate. $n=8$ per age group for each muscle; values are means \pm SEM; ${ }^{*} P<0.05$ vs. YA.

proving a role for intrinsic mitochondrial dysfunction in atrophy of aging muscle, and comparing muscles of contrasting fiber type could also clarify the role of fiber type in this process.

Impairments in mitochondrial respiratory capacity in muscle with aging are highly variable (Lanza \& Sreekumaran Nair, 2010), with a reduction seen in some studies (Cooper et al., 1992; Boffoli et al., 1994), but not in others (Hutter et al., 2007; Chabi et al., 2008). Furthermore, we have previously shown that mitochondrial isolation can exaggerate the degree of mitochondrial dysfunction with aging compared to permeabilized myofibers (Picard et al., 2010). The current study found no change in state III respiration in permeabilized myofibers after normalizing for myofiber bundle wet weight in fast-twitch Gas and EDL; however, the higher abundance of oxidative phosphorylation system subunits suggests that the intrinsic respiratory capacity is reduced in these fast muscles. As such, up-regulation of oxidative phosphorylation system protein content could be a compensatory mechanism to an intrinsic oxidative defect in fasttwitch muscle. On the other hand, a decrease in state III respiration was paralleled by a decrease in the abundance of oxidative phosphorylation system subunits and in CS within the slow-twitch Sol muscle. This suggests no change in intrinsic respiratory capacity in the Sol muscle, but rather a modest decline in muscle mitochondrial content with aging. The AL showed no change in either state III respiratory capacity or oxidative phosphorylation system subunit expression, despite a modestly reduced CS activity in this muscle. Thus, our data show that changes in muscle respiratory capacity are unrelated to atrophy with aging across these four muscles, but rather are largely fiber type-specific.

Another point of interest revealed by our results is that a decline in muscle mitochondrial content with aging, which has been suggested in some reports (Chabi et al., 2008), but not others (Mathieu-Costello et al., 2005; Figueiredo et al., 2009), depends on the muscle examined and is generally not a feature of aging muscles. The fact that there is no decline in mitochondrial content in fast muscle, despite the documented decline in peroxisome proliferator-activated receptor gamma coactivator 1-alpha (PGC-1 $\alpha$ ) in fast muscles like the Gas (Baker et al., 2006) and plantaris (Chabi et al., 2008) with aging, implicates reduced mitochondrial protein turnover in producing the respiratory dysfunction (unchanged respiratory capacity per mg of muscle mass, despite higher oxphos protein levels) we have observed in the fast muscles, as suggested previously (Baker et al., 2006).

Our results also contrast with data obtained with the mitochondrial DNA mutator mouse, a transgenic animal which possesses a proofreading deficient version of the mitochondrial polymerase- $\gamma$ (Hiona et al., 2010), and which exhibits a progeroid phenotype because of an accelerated accumulation of mitochondrial DNA damage (Trifunovic et al., 2004; Kujoth et al., 2005). Whereas this 'premature aging' model shows lower abundance of oxidative phosphorylation system proteins in a largely fast-twitch hindlimb muscle, we find that normally aging F344BN rats have higher abundance of oxidative phosphorylation system proteins with aging in the fast muscles. As such, our results suggest that the mechanisms driving muscle atrophy with normal aging are unlikely to be the result of accumulated mitochondrial DNA damage. On the other hand, our results in normally aging fasttwitch muscle are very similar to what is seen following surgical denervation where oxidative phosphorylation system subunits are also seen to be elevated in the fast-twitch tibialis anterior muscle (Abruzzo et al., 2010), suggesting denervation could be playing an important role in the aging mitochondrial phenotype.

The second mitochondrial function we examined was $\mathrm{H}_{2} \mathrm{O}_{2}$ emission as an indicator of mitochondrial ROS generation. Interestingly, $\mathrm{H}_{2} \mathrm{O}_{2}$ emission per $\mathrm{O}_{2}$ flux, which is a measure of free radical leak (Anderson \& Neufer, 2006), tended to be higher under state II conditions in the fast-twitch Gas and EDL, and the Gas exhibited a higher free radical leak under state III conditions. On the other hand, the slow-twitch AL muscle exhibited an increased free radical leak during state II conditions. As with the changes in respiration, the changes in $\mathrm{H}_{2} \mathrm{O}_{2}$ emission with aging did not parallel the different susceptibility to atrophy between muscles. Because endogenous scavengers of $\mathrm{H}_{2} \mathrm{O}_{2}$ are known to compete with the Amplex Red probe in this system of ROS measurement (Treberg et al., 2010), it is interesting to note the divergent changes in GPx and Cat activities with aging between these muscles. Specifically, GPx was elevated in the Gas and reduced in the Sol and AL, whereas Cat was elevated in both fast Gas and EDL, but lower in slow Sol and higher in slow AL. Collectively, these changes in endogenous $\mathrm{H}_{2} \mathrm{O}_{2}$ scavengers likely mean that the mitochondria from fast muscles do in fact exhibit an increase in ROS production with aging during state II conditions but that this is muted by an up-regulation of endogenous antioxidants. On the other hand, the elevated $\mathrm{H}_{2} \mathrm{O}_{2}$ emission seen in the slow AL muscle is likely a consequence of reduced endogenous $\mathrm{H}_{2} \mathrm{O}_{2}$ scavenging potential. Regardless of this point, the changes in $\mathrm{H}_{2} \mathrm{O}_{2}$ emission between muscles did not correspond to the differential atrophy observed, particularly for the AL muscle that actually hypertrophied by nearly $50 \%$ with aging. As elevated mitochondrial ROS are implicated in activation of muscle atrophy pathways like the proteasome (McClung et al., 2008), the changes we observed may not have been of sufficient magnitude to yield this effect. 

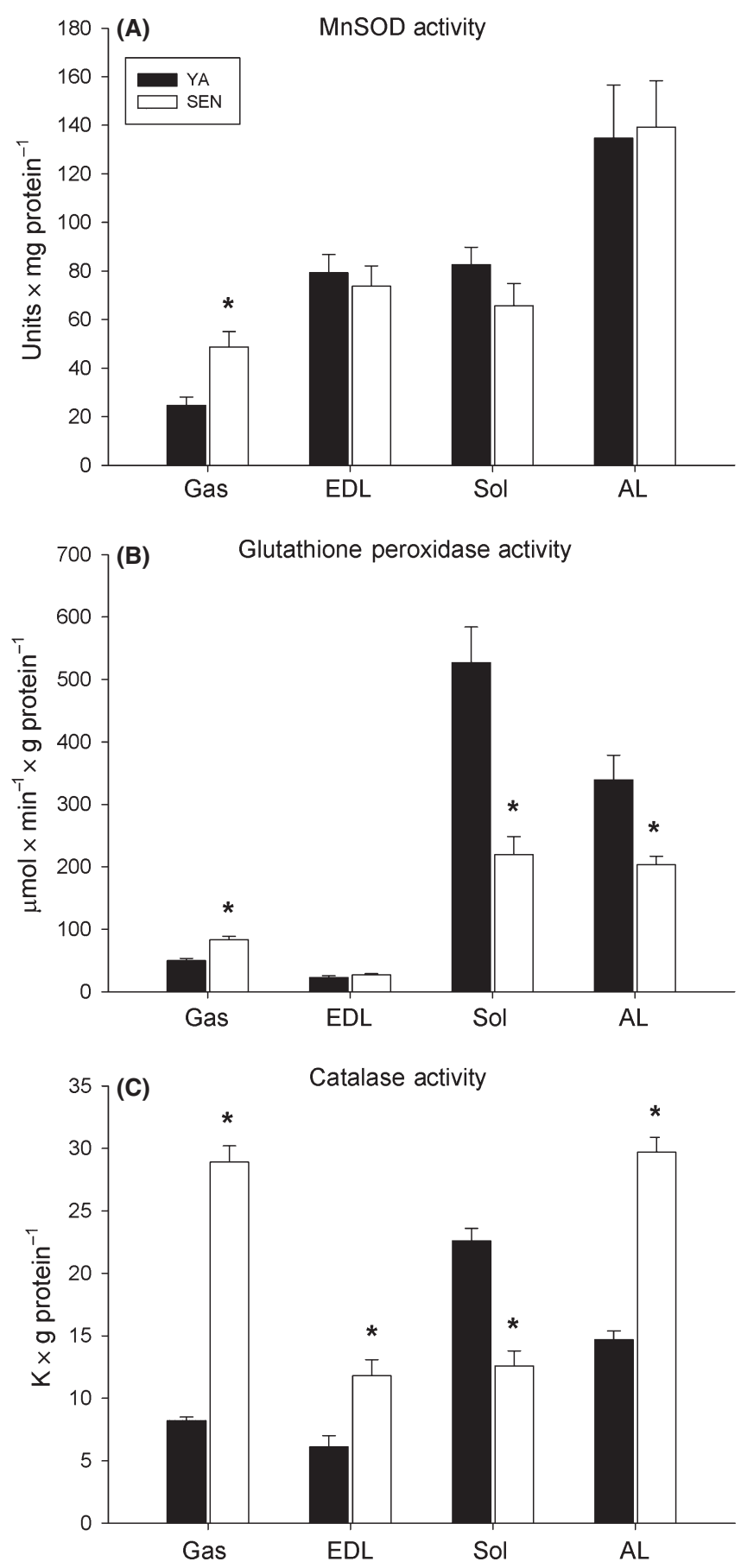

Fig. 5 Endogenous antioxidant activities measured on muscle homogenates of gastrocnemius (Gas), extensor digitorum longus (EDL), soleus (Sol), and adductor longus ( $\mathrm{AL}$ ) muscles of young adult (YA) and senescent (SEN) rats. (A) Manganese superoxide dismutase activity (MnSOD); (B) Glutathione peroxidase (GPx) activity; (C) Catalase activity. $n=8$ per age group for each muscle except $\mathrm{AL}$ (YA: $n=5$; SEN: $n=8)$; values are means \pm SEM; ${ }^{*} P<0.05$ vs. YA.

The final index of mitochondrial function that we examined was the sensitivity of the mPTP to a $\mathrm{Ca}^{2+}$ challenge. Given the abundance of studies finding elevated markers of apoptosis in aging muscles (Dirks \& Leeuwenburgh, 2002; Siu et al., 2005; Baker \& Hepple, 2006; Rice \& Blough, 2006; Chabi et al., 2008; Marzetti et al., 2008), our study helps clarify the role of intrinsic changes in the MPTP in this widely observed phenomenon. Overall, the changes in mPTP function did not correspond to the severity of muscle atrophy. Firstly, despite the Gas having nearly twice the amount of atrophy as the EDL, the changes in MPTP function were, if anything, greater in the EDL muscle. Secondly, despite the slow-twitch Sol and fast-twitch EDL exhibiting very similar degrees of atrophy with aging, only the EDL exhibited a change in MPTP function. In light of the evidence for an up-regulation of mitochondrial-mediated pathways of apoptosis in aging skeletal muscle (Chabi et al., 2008; Marzetti et al., 2008), our data suggests that this is unlikely to occur principally via an intrinsic change in susceptibility of the mPTP to opening, but rather secondary to other changes within the aged milieu, a point we discuss below.

\section{Significance of measures in permeabilized myofibers}

One of the primary benefits of using the permeabilized myofiber technique is the preservation of mitochondrial structure and content with this method (Saks et al., 1998; Picard et al., 2011). In addition, we have also found that routine mitochondrial isolation methods markedly exaggerate the degree of mitochondrial dysfunction in severely atrophied aging muscle (Picard et al., 2010). While these arguments provide a strong basis for using the permeabilized myofiber method, an important point of consideration is that the saponin-permeabilization of myofibers and subsequent incubation in standardized incubation buffers will result in a normalization of the intracellular milieu between age groups. As changes in the intracellular milieu are implicated in the dysfunction of many cell types with aging, e.g., myogenic precursor cells (Conboy et al., 2005), and cytoplasmic pro-apoptotic factors like Bax can influence mitochondrialdriven apoptotic pathways (Marzetti et al., 2010), it seems highly likely that soluble intracellular factors, including a wide array of pro-apoptotic signaling molecules that could impact mitochondria (e.g.,(Rice \& Blough, 2006; Marzetti et al., 2008), are washed out during the incubation of permeabilized myofibers.

Thus, while our data do not support a causal role for intrinsic mitochondrial dysfunction in atrophy of aging muscle, it is important to appreciate that our experiments represent the intrinsic function of the organelle under idealized conditions, and changes in the cellular environment with aging could still impact mitochondrial function in vivo. However, in this scenario, mitochondria do not have a primary defect that directly causes muscle atrophy, but instead are simply responding to changes in the aged milieu. This conceptual and functional distinction will be critical if we are to correctly identify the primary causes of sarcopenia and avoid chasing secondary targets, which our data suggests likely includes the mitochondrion.

\section{Experimental procedures}

\section{Animals and surgical methods}

All procedures were conducted with approval from the Animal Care Committee at University of Calgary (protocol ID BI09R-11). Male Fischer $344 \times$ Brown Norway F1-hybrid (F344BN) rats were obtained from the colony maintained by the National Institute on Aging (USA) at 810 months (YA) or 35-36 months (SEN). These ages are well documented to represent a period where significant age-related muscle atrophy and dysfunction exist (Hagen et al., 2004) and therefore allow one to examine factors related to sarcopenia. Animals were kept for at least $48 \mathrm{~h}$ following arrival at our institution before experiments (12:12 hour light/dark cycle, ambient temperature $23^{\circ} \mathrm{C}$ ). On experiment day, animals were anesthetized with 55-65 $\mathrm{mg} \mathrm{kg}^{-1}$ Sodium Pentobarbital (I.P.). 

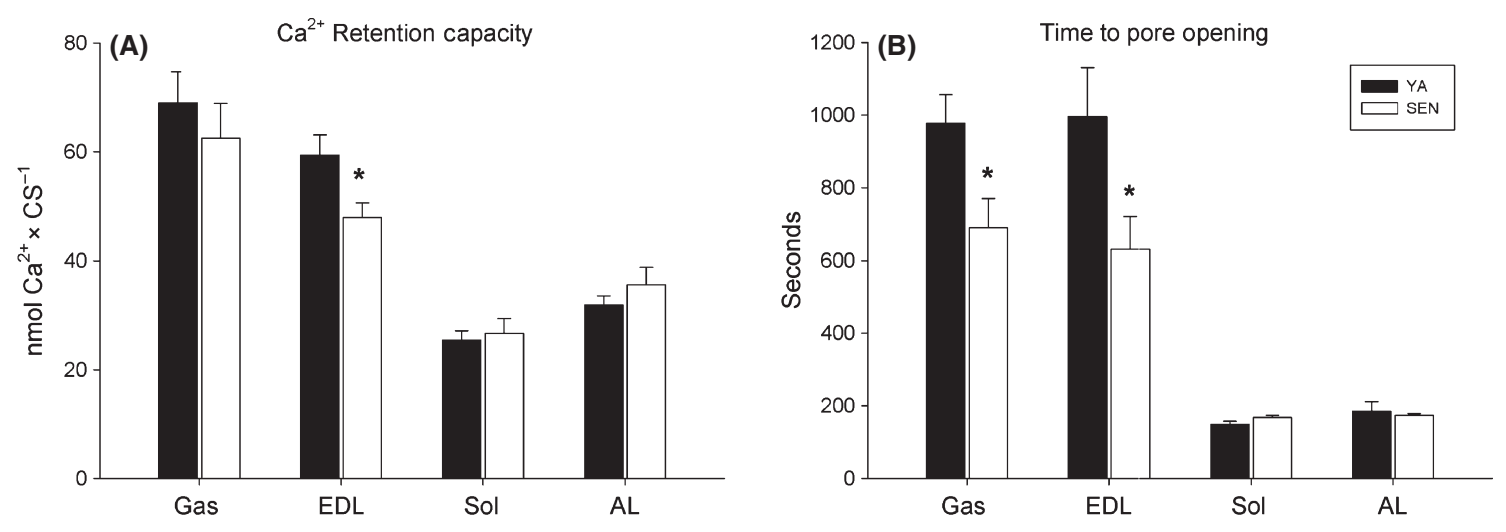

Fig. 6 Sensitivity of the mitochondrial permeability transition pore (mPTP) to a $20 \mu \mathrm{m} \mathrm{Ca}^{2+}$ challenge measured by following mitochondrial Ca ${ }^{2+}$ uptake with the fluorescent dye $\mathrm{Ca}^{2+}$ Green $5 \mathrm{~N}$ in permeabilized myofibers of gastrocnemius (Gas), extensor digitorum longus (EDL), soleus (Sol), and adductor longus (AL) muscles of young adult (YA) and senescent (SEN) rats. (A) $\mathrm{Ca}^{2+}$ retention capacity, normalized per international units of citrate synthase activity; (B) time to opening of the mPTP measured as described in the methods. $n=8$ per age group for each muscle; values are means $\pm \mathrm{SEM}$; ${ }^{*}<0.05$ vs. YA.

Bilateral dissection of four muscles was performed on eight $Y A$ and eight SEN animals: the predominantly fast-twitch EDL and mixed Gastrocnemius (Gas) muscles, and the predominantly slow-twitch Sol and AL muscles (Armstrong \& Phelps, 1984). Upon dissection, muscles were immediately put in ice-cold relaxing Buffer $A$ and weighed. Full details of experimental procedures, buffers and reagents are provided in (Picard et al., 2010). Note that the data for respiration, $\mathrm{H}_{2} \mathrm{O}_{2}$ emission and mPTP function of the Gas muscle have been presented previously (Picard et al., 2010).

\section{Abundance of electron transport chain complexes}

Muscle homogenates were prepared from each muscle of each animal, and $10 \mu \mathrm{g}$ of proteins was loaded into a precast 4-15\% SDS-polyacrylamide gels (SDS-PAGE) (Bio-Rad, Ontario, Canada). Proteins were transferred onto a membrane and incubated overnight with a premixed cocktail of polyclonal antibodies directed against representative subunits of each of the electron transport chain complexes (Mitosciences MS604, $6 \mu \mathrm{g} \mathrm{m}^{-1}$ ). Equal protein loading was verified using the Ponceau red stain. Membranes were washed in $0.05 \%$ Tween-PBS buffer, incubated with horseradish peroxidase-conjugated secondary antibody (dilution 1:1000), and bands densitometry was detected as previously described (Picard et al., 2010).

\section{Preparation of permeabilized myofibers}

Permeabilized myofiber bundles were prepared as previously described in (Picard et al., 2010). Briefly, whole muscles are manually dissected into small fiber bundles, and then incubated in saponin-supplemented $\left(0.05 \mathrm{mg} \mathrm{mL}^{-1}\right)$ relaxing buffer $A$ for $30 \mathrm{~min}$. Permeabilized myofibers were then rinsed in stabilizing Buffer $B$ and kept on ice until use for the different measurements. From day-to-day, the sequence of measurements on each of the four muscles was randomized to avoid an ordering effect on our results. All mitochondrial function measurements were performed at least in duplicates for each muscle.

\section{High-resolution respirometry}

Permeabilized myofibers were used to determine mitochondrial respiratory capacity under different activation states, using a polarographic oxygen sensor (Oxygraph-2k; Oroboros, Innsbruck, Austria). Briefly,
3.5-6 mg (wet weight) permeabilized bundles were added to the respiration chambers (one bundle in each of two the chambers) containing $2 \mathrm{~mL}$ of Buffer $\mathrm{B}$, at $37{ }^{\circ} \mathrm{C}$. Myofiber respiration was performed at hyperoxygenated levels to eliminate $\mathrm{O}_{2}$ diffusion limitations. State III respiration was measured with the addition of $10 \mathrm{~mm}$ glutamate $+2 \mathrm{~mm}$ malate $+10 \mathrm{~mm}$ succinate (GMS) and $2 \mathrm{~mm}$ adenosine di-phosphate (ADP). After respiration measurements were completed, bundles were frozen in liquid $\mathrm{N}_{2}$ and stored at $-80{ }^{\circ} \mathrm{C}$ for subsequent enzymatic measurements. Respiration was expressed as pmol $\mathrm{O}_{2} \mathrm{~s}-1 \times \mathrm{mg}^{-1}$ wet weight of individual permeabilized myofiber bundles and as pmol $\mathrm{O}_{2} \times$ min- 1 x enzymatic unit $(U)-1$ of $C S$ activity.

\section{Citrate synthase and cytochrome c oxidase activity}

Frozen myofiber bundles from respirometry assays were homogenized and used to detect spectrophotometrically the activity of CS and COX in all samples from YA and SEN animals, according to methods we have described previously (Picard et al., 2010).

\section{Mitochondrial $\mathrm{H}_{2} \mathrm{O}_{2}$ emission}

Mitochondrial ROS production was measured based on $\mathrm{H}_{2} \mathrm{O}_{2}$ emission from permeabilized myofibers, detected by the reaction in $\mathrm{H}_{2} \mathrm{O}_{2}$ with Amplex Red catalyzed by horseradish peroxidase as described in (Picard et al., 2010) and adapted from (Anderson \& Neufer, 2006). Briefly, 4-6 mg myofiber bundles were added to Buffer $Z$ at $37{ }^{\circ} \mathrm{C}$, to which were added $10 \mathrm{~mm}$ glutamate $+2 \mathrm{~mm}$ malate (State II, GM), then $10 \mathrm{~mm}$ succinate $+1 \mathrm{~mm}$ ADP (State III, GMS). Matching respiration values were used to compute $\mathrm{H}_{2} \mathrm{O}_{2}$ production per $\mathrm{O}_{2}$ flux. To compute $\mathrm{H}_{2} \mathrm{O}_{2}$ production per $\mathrm{O}_{2}$ flux for each muscle, we took the quotient of respiration and $\mathrm{H}_{2} \mathrm{O}_{2}$ emission for matched substrate conditions, where the respiration and $\mathrm{H}_{2} \mathrm{O}_{2}$ values had each been normalized to the CS activity measured in the individual bundles used in each experiment (different bundles were used in respiration vs. $\mathrm{H}_{2} \mathrm{O}_{2}$ experiments).

\section{Antioxidant enzymes activity}

Endogenous antioxidant enzyme activity was performed as we have described previously (Tweedie et al., 2011). Frozen tissue samples from each muscle weighing $15-30 \mathrm{mg}$ were homogenized at $4{ }^{\circ} \mathrm{C}$ and the 
resulting supernatant used in enzymatic assays of superoxide dismutase (SOD) before and after incubation with $\mathrm{NaCN}$ (to distinguish MnSOD from CuZnSOD), and in enzymatic assays of GPx and catalase activity.

\section{Mitochondrial permeability transition pore sensitivity to $\mathrm{Ca}^{2+}$}

Susceptibility of mPTP opening to $\mathrm{Ca}^{2+}$, a known pro-apoptotic event that happens in vivo, was measured in phantom permeabilized myofibers devoid of myosin, as described previously in (Picard et al., 2010). Then, 4-6 mg of phantom permeabilized myofibers were added to CRC buffer at $37^{\circ} \mathrm{C}$, supplemented with substrates (GM) that activate the electron transport chain and allow mitochondrial-specific $\mathrm{Ca}^{2+}$ intake. Extramitochondrial $\left[\mathrm{Ca}^{2+}\right]$ was monitored fluorometrically using the $\mathrm{Ca}^{2+}$ sensitive dye Calcium Green-5N (Molecular Probes, Invitrogen, Carlsbad, CA, USA) and used to determine CRC and time to MPTP opening as described in (Picard et al., 2010).

\section{Acknowledgments}

The authors wish to thank Ms. Sharon Rowan for her assistance in tissue collection, and Ms. Sharon Rowan and Ms. Warda Khan for conducting the antioxidant enzyme assays. This work was supported by operating grants from the Canadian Institutes for Health Research (MOP 57808 and IAO 84673 to RTH). MP is a CIHR fellow in Systems Biology and in Psychosocial Oncology and is supported by a scholarship from the Natural Sciences and Engineering Research council.

\section{Author contributions}

RTH designed the experiments, which were performed by MP, DR, KJW, and MMT. The data were analyzed by MP, RTH, and DR. RTH and MP interpreted the data and wrote the manuscript.

\section{References}

Abruzzo PM, di Tullio S, Marchionni C, Belia S, Fano G, Zampieri S, Carraro U, Kern H, Sgarbi G, Lenaz G, Marini M (2010) Oxidative stress in the denervated muscle. Free Radic. Res. 44, 563-576.

Anderson EJ, Neufer PD (2006) Type II skeletal myofibers possess unique properties that potentiate mitochondrial $\mathrm{H}(2) \mathrm{O}(2)$ generation. Am. J. Physiol. Cell Physiol. 290, C844-C851.

Armstrong RB, Phelps RO (1984) Muscle fiber type composition of the rat hindlimb. Am. J. Anat. 171, 259-272.

Baker DJ, Hepple RT (2006) Elevated caspase and AIF signaling correlates with the progression of sarcopenia during aging in male F344BN rats. Exp. Gerontol. 41, 1149-1156.

Baker DJ, Betik AC, Krause DJ, Hepple RT (2006) No decline in skeletal muscle oxidative capacity with aging in long-term caloric restricted rats: effects are independent of mtDNA integrity. J. Gerontol. A Biol. Sci. Med. Sci. 61A, 675684.

Boffoli D, Scacco SC, Vergari R, Solarino G, Santacroce G, Papa S (1994) Decline with age of the respiratory chain activity in human skeletal muscle. Biochim. Biophys. Acta 1226, 73-82.

Bua E, Johnson J, Herbst A, Delong B, McKenzie D, Salamat S, Aiken JM (2006) Mitochondrial DNA-deletion mutations accumulate intracellularly to detrimental levels in aged human skeletal muscle fibers. Am. J. Hum.Genet. 79, 469480.

Capel F, Rimbert V, Lioger D, Diot A, Rousset P, Mirand PP, Boirie Y, Morio B, Mosoni $L$ (2005) Due to reverse electron transfer, mitochondrial $\mathrm{H} 2 \mathrm{O} 2$ release increases with age in human vastus lateralis muscle although oxidative capacity is preserved. Mech. Ageing Dev. 126, 505-511.
Chabi B, Ljubicic V, Menzies KJ, Huang JH, Saleem A, Hood DA (2008) Mitochondrial function and apoptotic susceptibility in aging skeletal muscle. Aging Cell 7, 2-12.

Conboy IM, Conboy MJ, Wagers AJ, Girma ER, Weissman IL, Rando TA (2005) Rejuvenation of aged progenitor cells by exposure to a young systemic environment. Nature 433, 760-764.

Cooper JM, Mann VM, Schapira AH (1992) Analyses of mitochondrial respiratory chain function and mitochondrial DNA deletion in human skeletal muscle: effect of ageing. J. Neurol. Sci. 113, 91-98.

Cruz-Jentoft AJ, Landi F, Topinkova E, Michel JP (2010) Understanding sarcopenia as a geriatric syndrome. Curr. Opin. Clin. Nutr. Metab. Care. 13, 1-7.

Delp MD, Duan C (1996) Composition and size of type I, IIA, IID/X, and IIB fibers and citrate synthase activity of rat muscle. J. Appl. Physiol. 80, 261-270.

Desai VG, Weindruch R, Hart RW, Feuers RJ (1996) Influences of age and dietary restriction on gastrocnemius electron transport system activities in mice. Arch. Biochem. Biophys. 333, 145-151.

Dirks A, Leeuwenburgh C (2002) Apoptosis in skeletal muscle with aging. Am. J. Physiol. Regul. Integr. Comp. Physiol. 282, R519-R527.

Figueiredo PA, Powers SK, Ferreira RM, Amado F, Appell HJ, Duarte JA (2009) Impact of lifelong sedentary behavior on mitochondrial function of mice skeletal muscle. J. Gerontol. A Biol. Sci. Med. Sci. 64, 927-939.

Gouspillou G, Bourdel-Marchasson I, Rouland R, Calmettes G, Franconi JM, Deschodt-Arsac V , Diolez P (2010) Alteration of mitochondrial oxidative phosphorylation in aged skeletal muscle involves modification of adenine nucleotide translocator. Biochim. Biophys. Acta 1797, 143-151.

Hagen JL, Krause DJ, Baker DJ, Fu MH, Tarnopolsky MA, Hepple RT (2004) Skeletal muscle aging in F344BN F1-hybrid rats: I. Mitochondrial dysfunction contributes to the age-associated reduction in VO2max. J. Gerontol. A Biol. Sci. Med. Sci. 59, 1099-1110.

Hepple RT (2011) Alterations in mitochondria and their impact in aging skeletal muscle. In Sarcopenia - Age-related Muscle Wasting and Weakness: Mechanisms and Treatments (G Lynch, ed.). New York: Springer, pp. 135-158.

Hepple RT, Baker DJ, Kaczor JJ, Krause DJ (2005) Long-term caloric restriction abrogates the age-related decline in skeletal muscle aerobic function. FASEB J. 19, 1320-1322.

Hiona A, Leeuwenburgh C (2008) The role of mitochondrial DNA mutations in aging and sarcopenia: implications for the mitochondrial vicious cycle theory of aging. Exp. Gerontol. 43, 24-33.

Hiona A, Sanz A, Kujoth GC, Pamplona R, Seo AY, Hofer T, Someya S, Miyakawa T, Nakayama C, Samhan-Arias AK, Servais S, Barger JL, Portero-Otin M, Tanokura M, Prolla TA, Leeuwenburgh C (2010) Mitochondrial DNA mutations induce mitochondrial dysfunction, apoptosis and sarcopenia in skeletal muscle of mitochondrial DNA mutator mice. PLOS ONE 5, e11468.

Hutter E, Skovbro M, Lener B, Prats C, Rabol R, Dela F, Jansen-Durr P (2007) Oxidative stress and mitochondrial impairment can be separated from lipofuscin accumulation in aged human skeletal muscle. Aging Cell 6, 245-256.

Kujoth GC, Hiona A, Pugh TD, Someya S, Panzer K, Wohlgemuth SE, Hofer T, Seo AY, Sullivan R, Jobling WA, Morrow JD, Van Remmen H, Sedivy JM, Yamasoba T, Tanokura M, Weindruch R, Leeuwenburgh C, Prolla TA (2005) Mitochondrial DNA mutations, oxidative stress, and apoptosis in mammalian aging. Science 309, 481-484.

Lanza IR, Sreekumaran Nair K (2010) Regulation of skeletal muscle mitochondrial function: genes to proteins. Acta Physiol. (Oxf) 199(4), 529-547.

Ljubicic V, Hood DA (2009) Diminished contraction-induced intracellular signaling towards mitochondrial biogenesis in aged skeletal muscle. Aging Cell 8, 394404.

Marzetti E, Wohlgemuth SE, Lees HA, Chung HY, Giovannini S, Leeuwenburgh C (2008) Age-related activation of mitochondrial caspase-independent apoptotic signaling in rat gastrocnemius muscle. Mech. Ageing Dev. 129, 542-549.

Marzetti E, Hwang JC, Lees HA, Wohlgemuth SE, Dupont-Versteegden EE, Carter CS, Bernabei R, Leeuwenburgh C (2010) Mitochondrial death effectors: relevance to sarcopenia and disuse muscle atrophy. Biochim. Biophys. Acta 1800, 235-244.

Mathieu-Costello O, Ju Y, Trejo-Morales M, Cui L (2005) Greater capillary-fiber interface per fiber mitochondrial volume in skeletal muscles of old rats. J. Appl. Physiol. 99, 281-289.

McClung JM, Whidden MA, Kavazis AN, Falk DJ, Deruisseau KC, Powers SK (2008) Redox regulation of diaphragm proteolysis during mechanical ventilation. Am. J. Physiol. Regul. Integr. Comp. Physiol. 294, R1608-R1617.

Muller FL, Song W, Jang YC, Liu Y, Sabia M, Richardson A, Van Remmen H (2007) Denervation-induced skeletal muscle atrophy is associated with increased mitochondrial ROS production. Am. J. Physiol. Regul. Integr. Comp. Physiol. 293, R1159-1168. 
Navarro A, Boveris A (2007) The mitochondrial energy transduction system and the aging process. Am. J. Physiol. Cell Physiol. 292, C670-C686.

Picard M, Ritchie D, Wright KJ, Romestaing C, Thomas MM, Rowan SL, Taivassalo T, Hepple RT (2010) Mitochondrial functional impairment with aging is exaggerated in isolated mitochondria compared to permeabilized myofibers. Aging Cell 9, 1032-1046.

Picard M, Taivassalo T, Ritchie D, Wright KJ, Thomas MM, Romestaing C, Hepple RT (2011) Mitochondrial structure and function are disrupted by standard isolation methods. PLOS ONE 6, e18317.

Rice KM, Blough ER (2006) Sarcopenia-related apoptosis is regulated differently in fast- and slow-twitch muscles of the aging F344/NxBN rat model. Mech. Ageing Dev. 127, 670-679.

Rooyackers OE, Adey DB, Ades PA, Nair KS (1996) Effect of age on in vivo rates of mitochondrial protein synthesis in human skeletal muscle. Proc. Natl. Acad. Sci. U S A 93, 15364-15369.

Rowan SL, Purves-Smith FM, Solbak NM, Hepple RT (2011) Accumulation of severely atrophic myofibers marks the acceleration of sarcopenia in slow and fast twitch muscles. Exp. Gerontol. 46, 660-669.

Saks VA, Veksler VI, Kuznetsov AV, Kay L, Sikk P, Tiivel T, Tranqui L, Olivares J, Winkler K, Wiedemann F, Kunz WS (1998) Permeabilized cell and skinned fiber techniques in studies of mitochondrial function in vivo. Mol. Cell. Biochem. 184, 81-100.

Seo AY, Xu J, Servais S, Hofer T, Marzetti E, Wohlgemuth SE, Knutson MD, Chung HY, Leeuwenburgh C (2008) Mitochondrial iron accumulation with age and functional consequences. Aging Cell 7, 706-716.

Siu PM, Pistilli EE, Butler DC, Alway SE (2005) Aging influences cellular and molecular responses of apoptosis to skeletal muscle unloading. Am. J. Physiol. Cell Physiol. 288, C338-C349.

Tong JF, Yan X, Zhu MJ, Du M (2009) AMP-activated protein kinase enhances the expression of muscle-specific ubiquitin ligases despite its activation of IGF1/Akt signaling in C2C12 myotubes. J. Cell. Biochem. 108, 458-468.

Treberg JR, Quinlan CL, Brand MD (2010). Hydrogen peroxide efflux from muscle mitochondria underestimates matrix superoxide production - a correction using glutathione depletion. FEBS J 277(13), 2766-2778.
Trifunovic A, Wredenberg A, Falkenberg M, Spelbrink JN, Rovio AT, Bruder CE, Bohlooly Y, Gidlof S, Oldfors A, Wibom R, Tornell J, Jacobs HT, Larsson NG (2004) Premature ageing in mice expressing defective mitochondrial DNA polymerase. Nature 429, 417-423

Tweedie C, Romestaing C, Burelle Y, Safdar A, Tarnopolsky MA, Seadon S, Britton SL, Koch LG, Hepple RT (2011) Lower oxidative DNA damage despite greater ROS production in muscles from rats selectively bred for high running capacity. Am. J. Physiol. Regul. Integr. Comp. Physiol. 300, R544-553.

Wanagat J, Cao Z, Pathare P, Aiken JM (2001) Mitochondrial DNA deletion mutations colocalize with segmental electron transport system abnormalities, muscle fiber atrophy, fiber splitting, and oxidative damage in sarcopenia. FASEB J. 15, 322-332.

\section{Supporting Information}

Additional supporting information may be found in the online version of this article:

Fig. S1 Representative Western blot images for components of the oxidative phosphorylation system - subunits of the electron transport chain complexes I-IV and $F_{0} F_{1}$ ATP synthase.

Fig. S2 High-resolution oxygraphy results showing mitochondrial respiration normalized per mg wet weight in permeabilized myofiber bundles.

Fig. S3 Respiratory ratios measured in permeabilized myofiber bundles.

Fig. S4 Mitochondrial $\mathrm{H}_{2} \mathrm{O}_{2}$ release and response to exogenous ADP measured in permeabilized myofibers.

As a service to our authors and readers, this journal provides supporting information supplied by the authors. Such materials are peer-reviewed and may be re-organized for online delivery, but are not copy-edited or typeset. Technical support issues arising from supporting information (other than missing files) should be addressed to the authors. 